\title{
ANÁLISE DA SUSTENTABILIDADE NO MEIO RURAL: ESTUDOS PUBLICADOS NO BRASIL DE 2005 A 2014
}

\author{
Anderson Giovane Sontag \\ Mestrando em Administração \\ Universidade Estadual do Oeste do Paraná - Unioeste \\ andersonsontag@hotmail.com \\ Elizandra Da Silva \\ Professora adjunta no curso de Graduação e Mestrado Profissional em Administração \\ Doutorado em Administração \\ Universidade Estadual do Oeste do Paraná - Unioeste \\ elizandra.silva@unioeste.br \\ Elza Hofer \\ Doutorado em Administração \\ Professora no Mestrado Profissional em Administração \\ Universidade Estadual do Oeste do Paraná - Unioeste \\ elza_hofer@uol.com.br
}

\section{RESUMO}

O objetivo deste estudo é analisar as publicações sobre a sustentabilidade e sua mensuração no meio rural como uma aplicação prática, verificando a preferência dos autores por indicadores ou índices e se estes estão inseridos em modelos. Metodologicamente, trata-se de uma pesquisa documental, com uso da técnica de meta-análise, com abordagem quantitativa das publicações científicas e qualitativa por meio de estudos comparativos de publicações ou citações. A base é composta por 36 artigos publicados nos periódicos científicos indexados nas bases de dados SciELO e Spell que envolveram a temática de sustentabilidade e sua avaliação no meio rural. Os resultados apresentam uma evolução histórica crescente do tema, com uma preferência pelo uso de indicadores de sustentabilidade, analisando tanto o sistema produtivo quanto as comunidades rurais. Ainda, cinco artigos utilizaram modelos como Apoia-NovoRural, Pressão-Estado-Resposta (PER) e Dashboard of Sustainability na análise da sustentabilidade.

Palavras-chave: Indicadores; Índices; Sustentabilidade; Meio rural.

\section{ANALYSIS OF SUSTAINABILITY IN RURAL AREAS: STUDIES PUBLISHED IN BRAZIL FROM 2005 TO 2014}

\begin{abstract}
The objective of this study is to analyze publications on sustainability and its measurement in rural areas as a practical application, observing the preference of authors for indicators or indexes, included or not in the models. Methodologically, this paper is a documentary research, using metaanalysis technique, a quantitative approach of scientific publications, and qualitative comparative studies of publications or citations. The data is composed by 36 articles published in scientific journals indexed in SciELO and Spell databases about sustainability and evaluation in rural areas. The results show a growing historical evolution of the theme, with a preference for the use of sustainability indicators, analyzing both the production system and rural communities. Although, five articles used models as Apoia-NovoRural, Pressure-State-Response (PSR) and Dashboard of Sustainability for the analysis of sustainability.
\end{abstract}

Key words: Indexes; Indicators; Rural areas; Sustainability. 


\section{INTRODUÇÃO}

O conceito de desenvolvimento sustentável é o resultado da crescente consciência das ligações globais entre os problemas ambientais, questões socioeconômicas relacionadas com a pobreza e a desigualdade e as preocupações com um futuro saudável para a humanidade (Hopwood, Mellor, \& O'brien, 2005). A ideia de sustentabilidade, disposta na Agenda 21 Brasileira, permeia todas as dimensões da vida: econômica, social, territorial, científica e tecnológica, política e cultural, ligando o governo, o setor produtivo e a sociedade (CPDS, 2004). Assim, a sustentabilidade possui uma característica interdisciplinar. No campo do conhecimento específico ainda é fragmentada por não existir metamodelos capazes de sintetizar explicações gerais, fazendo com que cada campo da ciência entenda a sustentabilidade a seu modo, partindo de pressupostos particulares e considerando os compromissos explicativos e as formas de intervenção próprias de cada área do conhecimento (Callado \& Fensterseifer, 2009). Embora ocorram debates sobre a noção de sustentabilidade em quase todas as áreas do conhecimento, eles obrigatoriamente têm suas raízes nas reflexões de duas disciplinas chaves: ecologia e economia (Veiga, 2010).

As propostas de agricultura sustentável ainda são minoritárias e incipientes em certos contextos sociais da produção agrícola brasileira, mesmo se reconhecendo que em algumas regiões ocorrem avanços consideráveis nesta direção, com a implementação de políticas públicas de extensão, assistência técnica, pesquisa agrícola e de aporte de recursos financeiros em programas específicos para a produção agrícola sustentável (Assad \& Almeida, 2004). No meio rural, o exercício de práticas sustentáveis começa na pequena propriedade rural de cada agricultor familiar. Dessa forma, as ações locais influenciam o contexto global, na medida em que o indivíduo deve se enxergar como parte de um processo mais amplo, no qual suas ações, por menores que sejam, são responsáveis pelas alterações de qualidade de vida das gerações futuras (Kummer, 2007).

Encontrar maneiras de direcionar o desenvolvimento rural para formas mais sustentáveis, que atendam as exigências econômicas, sociais e ambientais constitui-se um grande desafio. $\mathrm{O}$ desenvolvimento não será sustentável caso algum aspecto não seja contemplado, assim, os indicadores de sustentabilidade, isolados ou integrados em marco ordenadores, constituem o instrumento de avaliação da sustentabilidade mais encontrado na literatura e usado empiricamente (Costa, 2010).

Neste contexto, o objetivo desta pesquisa é analisar as publicações sobre a sustentabilidade e sua forma de mensuração no meio rural, seja por indicadores ou índices de sustentabilidade. Considerando que a avaliação, a mensuração e o monitoramento da sustentabilidade requerem um conjunto de indicadores, é estatisticamente impensável fundir em um mesmo índice apenas duas de suas três dimensões (Veiga, 2010) e que "[...]sistemas de indicadores de desenvolvimento sustentável podem constituir uma importante ferramenta de gestão ambiental, pública ou privada, à medida que consigam orientar e mensurar o desenvolvimento" (Van Bellen, 2004, p.1). A questão que se propõe é verificar as pesquisas relacionadas à análise da sustentabilidade aplicada no meio rural e se esta ocorre por meio de indicadores ou índices de sustentabilidade.

Pesquisas de meta-análise ou de análise bibliométrica relacionadas à sustentabilidade, têm se destacado nos últimos anos, a exemplo de: Freitas, Souza, Teixeira e Jabbour (2013) pesquisaram a existência de artigos sobre gestão de recursos humanos e sustentabilidade nos principais periódicos da área de administração no Brasil com Qualis A1 a B3. Os autores indicaram que a relação entre essas duas áreas é recente no país, com as primeiras publicações em 2006, e dos 170 artigos, apenas nove apresentaram essa relação. Já Schimitt, Hayde e Dreher (2013) analisaram as publicações relacionadas à sustentabilidade como vantagem competitiva, por meio de estudo bibliométrico, na base ISI Web of Science buscando as principais características. Suas conclusões apresentam um aumento das publicações nos últimos anos e uma tendência a discussões em torno do equilíbrio entre a sustentabilidade e o desenvolvimento competitivo de organizações de setores variados.

O tema sustentabilidade e inovação foram analisados por Xavier, Lucca, Richter e Wittmann (2013) com um estudo bibliométrico sobre as publicações acerca da temática no período de 2002 a 
2012 na base ISI Web of Science. Os resultados apontam um progressivo aumento de publicações com seu ápice em 2011, com predominância do idioma inglês, sendo o tema considerado um hottopic [tópico destaque] no cenário internacional no período. Rocha, Gomes, Kneipp e Camargo (2013) analisaram as características das publicações relacionadas aos temas gestão de projetos e sustentabilidade na base ISI Web of Science buscando identificar as principais características das publicações e a identificação dos hot-topics relacionados.

Souza e Ribeiro (2013) investigaram o perfil das pesquisas e a evolução do tema sustentabilidade ambiental nos periódicos nacionais Qualis de Administração de A1 a B2, no período de 1992 a 2011. Destaca-se a pluralidade de subtemas e o crescimento de estudos quantitativos com relação às pesquisas qualitativas a partir de 2007. Luca, Cardoso, Vasconcelos e Pontes (2014) analisaram o tema sustentabilidade em pesquisas de administração com base nos anais do Encontro da Anpad (2003 a 2010) e da Revista de Gestão Social e Ambiental (2007 a 2010). Os resultados apontam uma elevada incidência de propostas de modelos.

Silinske, Marquetto, Grohmann, Battistella e Madruga (2014) analisaram as publicações em relação à sustentabilidade na área de economia empresarial na base ISI Web of Science no período de 2003 a 2012. Os resultados demonstraram uma evolução nas publicações com hot topics relacionados às dimensões da sustentabilidade: econômica, social e ambiental, bem como aspectos gerenciais das organizações. Szabo, Costa e Ribeiro (2014) buscaram analisar o estado da arte das publicações de artigos internacionais e nacionais, publicados de 1998 a 2011 relacionados à stakeholders e sustentabilidade. A base internacional utilizou ISI Web of Science e a nacional, o banco de dados de periódicos da Capes. Comparando os artigos internacionais com os nacionais, os autores observaram que há lacunas nas linhas: ética, geral, governança corporativa, responsabilidade corporativa e sustentabilidade. Os resultados também identificaram lacunas na pesquisa internacional a partir de temas estudados no Brasil, tais como governança corporativa e desempenho, responsabilidade corporativa e políticas públicas na sustentabilidade.

Diversas entidades acadêmicas e políticas possuem interesse no desenvolvimento dos indicadores já existentes e no surgimento de novas propostas que sejam adaptadas às particularidades de suas realidades (Callado \& Fensterseifer, 2009). Essa pesquisa busca o acompanhamento dos estudos com aplicações práticas, que pretendem mensurar o nível de sustentabilidade das comunidades ou de sistemas produtivos, permitindo aos pesquisadores uma análise preliminar de experiências que melhor se adaptem as suas realidades. A lacuna teórica está na estruturação da análise do nível de sustentabilidade, dividida basicamente em dois grupos: indicadores e índices. Estes, por vezes, inseridos em marco ordenadores.

\section{FUNDAMENTAÇÃO TEÓRICA}

Esta seção traz a base teórica da pesquisa dividida em três subseções: indicadores, índices e marco ordenadores. $\mathrm{O}$ termo indicador é um parâmetro analisado isoladamente ou combinado com outros para refletir sobre as condições do sistema em análise; o termo índice é entendido como um valor numérico que representa a correta interpretação da realidade de um sistema simples ou complexo (natural, econômico ou social) utilizando em seu cálculo métodos ou bases científicas adequadas. O índice pode servir como um instrumento para tomada de decisão ou previsão e é considerado um nível superior da junção de um grupo de indicadores ou variáveis (Siche, Agostinho, Ortega, \& Romeiro, 2007).

\subsection{Indicadores de sustentabilidade}

O principal objetivo de um indicador é o de agregar e quantificar informações de uma maneira que sua significância fique mais aparente, simplificando as informações sobre fenômenos complexos, tentando melhorar o processo de comunicação (Van Bellen, 2004). Um bom indicador precisa ser confiável, útil e não ser caro na relação custo versus benefício. Precisa, ainda, tratar de

Revista de Gestão Social e Ambiental - RGSA, São Paulo, v. 10, n. 2, p. 70-86, maio/ago. 2016. 
um tema relevante, ter base teórica (validade), ter uma boa cobertura estatística (em termos regionais e seus componentes), ser sensível e específico com relação às mudanças do objeto que está sendo mensurado, ser de fácil entendimento para o público especializado (inteligibilidade de sua construção) e para o público em geral (comunicação), periodicamente atualizável, desagregável em suas partes e ter uma série histórica (Carvalho \& Barcelos, 2009).

Os indicadores de sustentabilidade requerem uma visão integrada do mundo com indicadores multidimensionais que mostrem as inter-relações existentes entre si. Diferente dos indicadores tradicionais de progresso econômico, social e ambiental, pois medem apenas as mudanças de um aspecto como se fossem inteiramente independentes dos demais (Callado e Fensterseifer, 2009).

Em relação aos indicadores de sustentabilidade, destacam-se os princípios de Bellagio, que norteiam a construção dos indicadores em dez pontos (Hardi \& Zdan, 1997; Carvalho \& Barcelos, 2009): (1) orientar a visão e os objetivos, a avaliação dos progressos em direção ao desenvolvimento sustentável; (2) perspectiva holística, incluindo a revisão de todo o sistema, bem como suas partes, considerando o bem-estar dos subsistemas social, econômico e ambiental; (3) elementos essenciais, considerando a equidade e a disparidade que há entre a população atual e as gerações passadas e futuras, que lidam com essas preocupações, tais como utilização de recursos, excesso de consumo e da pobreza, os direitos humanos e o acesso aos serviços, considerando as condições ecológicas das quais a vida depende; e considerando o desenvolvimento econômico e outras atividades não mercantis que contribuem para o bem estar humano social; (4) margem adequada, adotando um horizonte de tempo longo suficiente para capturar tanto as ações humanas como ecossistemas, permitindo a tomada de decisão a curto e longo prazo; (5) enfoque prático, com um conjunto explícito de categorias ou um sistema organizado que liga as visões, metas para os indicadores e critérios de avaliação, com a padronização da medida para permitir a comparação dos valores de indicadores de metas, referência, intervalos, limiares ou direção de tendências, conforme o caso; (6) abertura, permitindo tornar os métodos e dados que são usados acessíveis a todos; (7) comunicação eficaz, sendo projetado para atender às necessidades do público; (8) ampla participação, obtendo a ampla representação das bases fundamentalistas, profissionais, técnicas e sociais, incluindo grupo de jovens, mulheres, indígenas, garantindo o reconhecimento de valores diversos e dinâmicos; (9) avaliação contínua, desenvolvendo a capacidade de repetidas medidas para determinar tendências, sendo iterativo, adaptável, sensível às mudanças e incertezas, promovendo o aprendizado coletivo e o feedback; (10) capacidade institucional, atribuindo claramente às responsabilidades, prestação de apoio durante o processo, fornecendo capacidade institucional para a coleta de dados, manutenção e documentação, apoiando o desenvolvimento da capacidade de avaliação local.

Pode-se apontar uma série de características dos indicadores de sustentabilidade, tais como (1) seletividade, pois os indicadores devem estar relacionados a fatores essenciais ou críticos do processo a ser avaliado; (2) possuir representatividade, devendo ser escolhidos ou formulados de forma que possam representar satisfatoriamente o processo ou produto a que se refere; (3) simplicidade, ou seja, de fácil compreensão e aplicação, principalmente para aquelas pessoas diretamente envolvidas com a coleta, processamento e avaliação dos dados, requerendo o mínimo de esforço adicional para sua implementação; (4) ser de baixo custo, considerando que o custo para coleta, processamento e avaliação não devem ser superiores ao benefício trazido pela medida; (5) estabilidade, devendo ser coletados com base em procedimentos estruturados, incorporados às atividades da empresa que permitam sua comparação ou análise de tendência ao longo do tempo; (6) permitir a comparação externa, alguns indicadores devem ser desenvolvidos para permitir a comparação do desempenho da empresa com o de outras empresas de outros setores; (7) apresentar uma melhoria contínua, pois os indicadores devem ser periodicamente avaliados e, quando necessário, devem ser modificados ou ajustados para atender às mudanças no ambiente organizacional sem perder seu propósito e validade (Berliner \& Brimson, 1988; Tironi, Silva, 
Viana, \& Medici, 1991; Neely, Richards, Mills, Platts, \& Bourne, 1997; Callado e Fensterseifer, 2009).

\section{2 Índices de sustentabilidade}

Em uma análise superficial, índice e indicador possuem o mesmo significado, porém o índice é o valor agregado final de todo o procedimento de cálculo onde se utilizam, inclusive, indicadores como variáveis que o compõem (Siche et al., 2007). Na elaboração de um índice de sustentabilidade, um ponto crítico é a metodologia adotada, tanto para sua determinação, quanto para sua leitura e interpretação. A escolha deve ser clara e transparente, não deve deixar dúvidas sobre quais os princípios que estão na base do processo. Não é possível determinar sua sustentabilidade considerando apenas um aspecto do sistema, mas sim, pelo conjunto de fatores (econômicos, sociais e ambientais) que devem ser contemplados no cálculo do índice de sustentabilidade pelos correspondentes indicadores (Bouni, 1996, apud Siche et al., 2007).

O índice de sustentabilidade implica na explicação dos mecanismos e das lógicas atuantes na área sob análise e na quantificação dos fenômenos mais importantes que ocorrem no sistema. Por meio destes dois itens, é possível conhecer como a ação humana está afetando seu entorno, alertar sobre os riscos de sobrevivência humana e animal, prever situações futuras e guiar a tomada de decisão das políticas públicas (Siche et al., 2007).

Os índices ou indicadores dão uma fotografia do momento atual, funcionado como sinal de alarme para manifestar a situação do sistema avaliado, pois são valores estáticos. Os indicadores são normalmente utilizados como pré-tratamento aos dados originais e os índices correspondem a um nível superior de agregação (Siche et al., 2007). Alguns exemplos de índices de sustentabilidade são: Ecological Footprint ou Pegada Ecológica (PE), Environmental Sustentability Index ou Índice de Sustentabilidade Ambiental (ISA) e o Índice de Progresso Genuíno (IPG).

\subsection{Marcos ordenadores}

Os marcos metodológicos ou marcos ordenadores são desenvolvimentos teóricos que propõem estruturas analíticas flexíveis, para fundamentar o processo de análise da sustentabilidade de uma atividade econômica, incluindo as etapas de seleção, desenho e interpretação de indicadores, assim como a organização dos dados e a comunicação dos resultados finais. Dessa forma, os marcos contribuem para constituir a base lógica que permite guiar o processo de análise da sustentabilidade, propiciando a geração de resultados que permitem orientar o desenvolvimento de políticas e programas de promoção da sustentabilidade (Sanches \& Matos, 2012). Os marcos metodológicos de avaliação da sustentabilidade podem ser classificados em analíticos, sistêmicos e normativos.

Os marcos analíticos são baseados em modelos causais, ou seja, um processo analítico que procura identificar as relações de causa e efeito do sistema analisado. Parte do pressuposto de que o sistema ambiental provê recursos naturais para o processo de produção, também assimila os dejetos da produção e do consumo. O paradigma que rege essa interação é o uso racional de tais recursos naturais em um modelo de estímulo-estado-resposta, ou seja, um modelo casual. Como limitações a estes marcos têm-se a impossibilidade de estabelecer valores limites dos indicadores mediante critérios científicos ecológicos, a existência de fatores exógenos físicos e biológicos inesperados e de difícil previsão que exerçam influência sobre o sistema, ou a identificação das complexas relações ecológicas entre as espécies (Sanches \& Matos, 2012). Como exemplos de marcos metodológicos analíticos podem ser citados: Pressão - Estado - Resposta (PER) (Organization for Economic Cooperation and Development [OCDE], 1993) e Driving Force Pressures Impacts Responses (Dpsir) (Agência Europeia do Ambiente [AEA], 1995). O Modelo Pressão Estado Resposta (PER) possui três variantes: o modelo Força Motriz Estado Resposta (FER) substitui a Pressão pela Força Motriz (F), este adotado pela Comissão de Desenvolvimento Sustentável das 
Nações Unidas, em 1995; o Modelo Pressão Estado Impacto Resposta (Peir) inclui o Impacto (I) e é utilizado pelo Programa das Nações Unidas para o Meio Ambiente [Unep]; já o Modelo Força Motriz Estado Impacto Resposta (Peir), inclui a Força Motriz (F) Impacto (I) no PER (Carvalho \& Barcelos, 2009).

Os marcos sistêmicos são baseados em modelos que permitem uma interpretação sistêmica. O pressuposto de base é que a complexidade ambiental não permite ao ser humano entender o funcionamento dos sistemas em sua totalidade. Esta ignorância e incerteza levam à priorização de regras práticas, ou seja, ao invés de realizar medições exatas, busca-se identificar os princípios gerais (ou atributos de caráter fundamental) dos ecossistemas, assim como os respectivos impactos humanos mediante mapas sistêmicos, empregando modelos de relações sistêmicas (Sanches \& Matos, 2012). Como exemplos de marcos sistêmicos têm-se: o marco Dashboard of Sustainability (Hardi \& Zdan, 2000) e o Apoia Novo-Rural (Rodrigues \& Campanhola, 2003).

Os marcos normativos são baseados em modelos hierárquicos, dos quais os objetivos para alcançar um conteúdo disciplinar específico apresentam-se listados de forma hierárquica. Sob este enfoque, o conceito de sustentabilidade requer uma abordagem multidimensional, considerando aspectos ambientais, econômicos e sociais. A opção de analisar a sustentabilidade em dimensões consiste em uma aproximação normativa que permite definir objetivos ou metas de sustentabilidade para setores econômicos ou unidades produtivas. A vantagem é seu aspecto participativo, que permite o intercâmbio de informações entre os atores envolvidos. As estratégias de mudanças propostas por estes marcos costumam ser plasmadas sob recomendações políticas para o manejo e a distribuição de recursos. Os indicadores são derivados tanto das relações causa-efeito típica dos marcos analíticos, bem como do estabelecimento de princípios gerais de sustentabilidade identificados a partir de análise sistêmica. Conjuntamente com o marco analítico, o marco normativo é um dos mais empregados na literatura de indicadores de sustentabilidade (Sanches \& Matos, 2012). Como exemplos de marcos metodológicos normativos têm-se: o marco Marco de Evalución de Sistemas de Manejo de Recursos Naturales incorporando indicadores de sustentabilidad (Mesmis) (Masera, Astier, \& Lopez-Ridaura, 1999) e indicateurs de durabilité des exploitations agricoles (Idea) (Briquel, Vilain, Bourdais, Girardin, Mouchet, \& Viaux, 2001).

\section{MÉTODO DE PESQUISA}

Este estudo classifica-se como pesquisa descritiva, estabelecendo relações entre variáveis de uma determinada população (Gil, 2010; Raupp e Bauren, 2012). A tipologia quanto à natureza foi conceituada como uma pesquisa aplicada, por objetivar a geração de conhecimentos para aplicação prática, dirigidos à solução de problemas específicos, envolvendo verdades e interesses locais (Gil, 2010). Na abordagem do problema, a pesquisa pode ser classificada como: quantitativa das publicações científicas de um pesquisador ou instituição em periódicos com seleção arbitrada; também qualitativa por meio de estudos comparativos de publicações ou citações (Souza \& Ribeiro, 2013).

Quanto ao procedimento técnico, este foi qualificado como documental, que conforme Raupp e Bauren (2012, p. 89), "[...]baseia-se em materiais que ainda não receberam um tratamento analítico ou que podem ser reelaborados de acordo com os objetivos da pesquisa[...]", organizando informações que se encontram dispersas e conferindo-lhe uma nova importância como fonte de consulta. Utilizando-se de técnicas meta-análise (Melo \& Andreassi, 2010; Souza \& Ribeiro, 2013) busca-se analisar o perfil das publicações científicas com aplicação prática destinada a mensuração da sustentabilidade rural por indicadores ou índices, inseridos em modelos ou não.

O planejamento da pesquisa passou por quatro etapas: (1) a escolha da literatura analisada, (2) a avaliação dos dados coletados, (3) a análise e interpretação das informações e (4) a apresentação dos resultados, conforme sugerem Cooper \& Lindsay (1998) e Souza \& Ribeiro (2013). 
A perspectiva temporal contempla trabalhos publicados entre os períodos de 2005 a 2014, ou seja, últimos 10 anos. A coleta de dados baseia-se nos artigos publicados nos periódicos científicos indexados nas bases de dados da Scientific Eletronic Library Online [SciELO] e Scientific Periodicals Eletronic Library [Spell], sendo estes as fontes primárias da pesquisa realizada na primeira quinzena de abril/2015. Obteve-se 36 artigos de acordo com os critérios de busca, sendo apresentados na figura 1 os autores e títulos analisados na pesquisa. A escolha das bases SciELO e Spell se justificam pela interdisciplinaridade do tema sustentabilidade aplicado ao meio rural, uma vez que pode abordar diferentes estratos da Qualis Capes.

A busca aconteceu pelos termos "sustentabilidade + indicadores", "sustentabilidade + índice" e "sustentabilidade + modelo", inseridos em todos os critérios de busca disponíveis nas bases. Após a seleção dos artigos, realizou-se a leitura dos resumos, metodologia e conclusão para enquadramento ao objetivo da pesquisa. Em alguns casos, houve a necessidade da leitura completa dos trabalhos para melhor compreensão. Em seguida, os trabalhos foram organizados em planilhas no software Excel para formatação e análise.

\begin{tabular}{|c|c|c|}
\hline Autor/Data & Título & Base \\
\hline Romanelli e Milan (2005) & $\begin{array}{l}\text { Energy balance methodology and modeling of supplementary forage } \\
\text { production for cattle in brazil }\end{array}$ & SciELO \\
\hline Barreto, Khan e Lima (2005) & Sustentabilidade dos assentamentos no município de Caucaia - CE & SciELO \\
\hline $\begin{array}{l}\text { Campos, Saglietti, Campos e } \\
\text { Bueno (2005) }\end{array}$ & Análise energética na produção de feno de cynodon dactylon (l.) Pers & SciELO \\
\hline Andrade (2007) & $\begin{array}{l}\text { Indicadores de sustentabilidade na reserva de desenvolvimento sustentável } \\
\text { da piranha, Manacapuru, Amazonas, Brasil. }\end{array}$ & SciELO \\
\hline Ribas, Severo e Miguel (2007) & $\begin{array}{l}\text { Agricultura familiar, extrativismo e sustentabilidade: o caso dos } \\
\text { "samambaieiros" do litoral norte do Rio Grande do Sul }\end{array}$ & SciELO \\
\hline Finatto e Salamoni (2008) & $\begin{array}{l}\text { Agricultura familiar e agroecologia: perfil da produção de base } \\
\text { agroecológica do município de Pelotas - RS }\end{array}$ & SciELO \\
\hline $\begin{array}{l}\text { Neto, Andrade, Rosa, Mota e } \\
\text { Lopes (2008) }\end{array}$ & $\begin{array}{l}\text { Índice de sustentabilidade agroambiental para o perímetro irrigado Ayres } \\
\text { de Souza }\end{array}$ & SciELO \\
\hline Araujo e Sá (2008) & $\begin{array}{l}\text { Sustentabilidade da piscicultura no baixo São Francisco alagoano: } \\
\text { condicionantes socioeconômicos }\end{array}$ & SciELO \\
\hline $\begin{array}{l}\text { Souza, Andrade e Cândido } \\
(2008)\end{array}$ & $\begin{array}{l}\text { A aplicação das dimensões do desenvolvimento sustentável: um estudo } \\
\text { exploratório nos municípios produtores de leite bovino no estado da } \\
\text { Paraíba }\end{array}$ & Spell \\
\hline $\begin{array}{l}\text { Gomes, Mello e Mangabeira } \\
(2009)\end{array}$ & $\begin{array}{l}\text { Estudo da sustentabilidade agrícola em um município amazônico com } \\
\text { análise envoltória de dados }\end{array}$ & SciELO \\
\hline $\begin{array}{l}\text { Vernetti Junior, Gomes e } \\
\text { Schuch (2009) }\end{array}$ & $\begin{array}{l}\text { Sustentabilidade de sistemas de rotação e sucessão de culturas em solos de } \\
\text { várzea no sul do Brasil }\end{array}$ & SciELO \\
\hline $\begin{array}{l}\text { Jasper, Biaggioni, Silva, Seki } \\
\text { e Bueno (2010) }\end{array}$ & $\begin{array}{l}\text { Análise energética da cultura do crambe (crambe abyssinica hochst) } \\
\text { produzida em plantio direto }\end{array}$ & SciELO \\
\hline Carvalho e Oliveira (2010) & $\begin{array}{l}\text { Economic feasibility of timber management in extractive settlement } \\
\text { projects of southwestern Amazonia }\end{array}$ & SciELO \\
\hline $\begin{array}{l}\text { Pereira, Lino, Buschinelli, } \\
\text { Barroes e Rodrigues (2010) }\end{array}$ & $\begin{array}{l}\text { Integrated farm environmental management and biodiversity conservation: } \\
\text { a case study in the caratinga biological station (Minas Gerais State, Brazil) }\end{array}$ & SciELO \\
\hline $\begin{array}{l}\text { Damasceno, Kahn e Lima } \\
\text { (2011) }\end{array}$ & $\begin{array}{l}\text { O impacto do Pronaf sobre a sustentabilidade da agricultura familiar, } \\
\text { geração de emprego e renda no estado do Ceará }\end{array}$ & SciELO \\
\hline $\begin{array}{l}\text { Santos, Mota, Santos, } \\
\text { Monteiro e Fontenele (2011) }\end{array}$ & $\begin{array}{l}\text { Avaliação da sustentabilidade ambiental do uso de esgoto doméstico } \\
\text { tratado na piscicultura }\end{array}$ & SciELO \\
\hline $\begin{array}{l}\text { Pugliesi, Marinho, Marques e } \\
\text { Lucarelli (2011) }\end{array}$ & $\begin{array}{l}\text { Valoração econômica do efeito da erosão em sistemas de manejo do solo } \\
\text { empregando o método custo de reposição }\end{array}$ & SciELO \\
\hline
\end{tabular}




\begin{tabular}{|c|c|c|}
\hline $\begin{array}{l}\text { Meireles, Araujo Neto e } \\
\text { Oliveira (2011) }\end{array}$ & Sustentabilidade do modelo agrícola da bacia do riacho Faé & SciELO \\
\hline Alves e Bastos (2011) & $\begin{array}{l}\text { Sustentabilidade em Silvânia (GO): o caso dos assentamentos rurais São } \\
\text { Sebastião da Garganta e João de Deus }\end{array}$ & SciELO \\
\hline Lima, Doria e Freitas (2012) & $\begin{array}{l}\text { Pescarias artesanais em comunidades ribeirinhas na Amazônia brasileira: } \\
\text { perfil socioeconômico, conflitos e cenário da atividade. }\end{array}$ & SciELO \\
\hline Rodrigues e Najberg (2012) & $\begin{array}{l}\text { Indicadores de sustentabilidade das políticas públicas decorrentes da } \\
\text { expansão do setor sucroalcooleiro em Carmo do Rio Verde (GO) }\end{array}$ & Spell \\
\hline $\begin{array}{l}\text { Poerch, Milach, Cavalli, } \\
\text { Wasielesky Jr, Möller e } \\
\text { Castello (2013) }\end{array}$ & $\begin{array}{l}\text { Use of a mathematical model to estimate the impact of shrimp pen culture } \\
\text { at Patos Lagoon Estuary, Brazil }\end{array}$ & SciELO \\
\hline $\begin{array}{l}\text { Eurich, Weirich Neto e Rocha } \\
\text { (2013) }\end{array}$ & $\begin{array}{l}\text { Índices emergéticos de sustentabilidade da produção leiteira em uma } \\
\text { propriedade de base familiar em Palmeira, Paraná, Brasil }\end{array}$ & SciELO \\
\hline Capellesco e Cazella (2013) & $\begin{array}{l}\text { Indicador de sustentabilidade dos agroecossistemas: estudo de caso em } \\
\text { áreas de cultivo de milho }\end{array}$ & SciELO \\
\hline $\begin{array}{l}\text { Araujo, Brandão Junior e } \\
\text { Colen (2013) }\end{array}$ & $\begin{array}{l}\text { Energetic analysis of landrace varieties and hybrids of corn produced in } \\
\text { different technological levels of management }\end{array}$ & SciELO \\
\hline $\begin{array}{l}\text { Rodrigues Filho e Juliani } \\
(2013)\end{array}$ & $\begin{array}{l}\text { Sustentabilidade da produção de etanol de cana-de-açúcar no estado de } \\
\text { São Paulo }\end{array}$ & SciELO \\
\hline $\begin{array}{l}\text { Silva, Barbosa e Albuquerque } \\
(2013)\end{array}$ & $\begin{array}{l}\text { Sustentabilidade de empreendimentos econômicos solidários: análise da } \\
\text { cooperativa dos fruticultores de Abaetetuba }\end{array}$ & $\begin{array}{l}\text { SciELO / } \\
\text { Spell }\end{array}$ \\
\hline Santos e Cândido (2013) & $\begin{array}{l}\text { Sustentabilidade e agricultura familiar: um estudo de caso em uma } \\
\text { associação de agricultores rurais }\end{array}$ & Spell \\
\hline $\begin{array}{l}\text { Baretta, Bartz, Fachini, } \\
\text { Anselmi, Zortéa e Baretta } \\
(2014)\end{array}$ & $\begin{array}{l}\text { Soil fauna and its relation with environmental variables in soil } \\
\text { management systems }\end{array}$ & SciELO \\
\hline $\begin{array}{l}\text { Santos, Siqueira, Araújo e } \\
\text { Maia (2014) }\end{array}$ & $\begin{array}{l}\text { A agroecologia como perspectiva de sustentabilidade na agricultura } \\
\text { familiar }\end{array}$ & SciELO \\
\hline Vidal e Santos (2014) & $\begin{array}{l}\text { Sustentabilidade rural no semiárido cearense: uma análise social, biofísica } \\
\text { e microeconômica em comunidades rurais }\end{array}$ & Spell \\
\hline $\begin{array}{l}\text { Ramos, Campos, Júnior e } \\
\text { Silva (2014) }\end{array}$ & $\begin{array}{l}\text { Sustentabilidade na produção de leite: balanço energético em sistema } \\
\text { intensivo de produção com visão focada nos processos }\end{array}$ & SciELO \\
\hline $\begin{array}{l}\text { Gomes, Peruzatto, Santos e } \\
\text { Sellitto (2014) }\end{array}$ & Indicadores de sustentabilidade na avaliação de granjas suinícolas & SciELO \\
\hline $\begin{array}{l}\text { Cardoso, Souza, Paiva, Momo } \\
\text { e Cunha (2014) }\end{array}$ & $\begin{array}{l}\text { Desenvolvimento territorial sustentável: estudo comparativo de } \\
\text { indicadores do sistema de gestão estratégica em territórios rurais do Rio } \\
\text { Grande do Norte }\end{array}$ & Spell \\
\hline $\begin{array}{l}\text { Ferreira, Neumann e } \\
\text { Hoffmann (2014) }\end{array}$ & $\begin{array}{l}\text { Análise da matriz energética e econômica das culturas de arroz, soja e } \\
\text { trigo em sistemas de produção tecnificados no Rio Grande do Sul }\end{array}$ & SciELO \\
\hline Borges e Borges (2014) & $\begin{array}{l}\text { Gestão regional e sustentabilidade energética: uma análise de indicadores } \\
\text { no setor agropecuário paraense }(2002-2010)\end{array}$ & Spell \\
\hline
\end{tabular}

\section{Figura 1 - Relação dos artigos por autor e título}

Fonte: Dados da pesquisa (2015)

\section{ANÁLISE E DISCUSSÃO DOS RESULTADOS}

A pesquisa obteve o resultado de 36 artigos enquadrados nos critérios metodológicos propostos, observa-se uma evolução do tema relacionado à sustentabilidade aplicada ao meio rural no período 2005 a 2014, com destaque para 2013 e 2014 (Figura 2). 


\begin{tabular}{|c|c|c|c|c|c|c|c|c|c|c|c|}
\hline Período & 2005 & 2006 & 2007 & 2008 & 2009 & 2010 & 2011 & 2012 & 2013 & 2014 & Total \\
\hline Artigos & 3 & 0 & 2 & 4 & 2 & 3 & 5 & 2 & 7 & 8 & 36 \\
\hline
\end{tabular}

Figura 2 - Evolução das publicações científicas ao longo do tempo

Fonte: Dados da pesquisa (2015)

A evolução histórica crescente do tema sustentabilidade também é característica presente em outros estudos de meta-análise, tais como os de Schimitt, Hayde e Dreher (2013), Xavier, Lucca, Richter e Wittmann (2013), Souza e Ribeiro (2013), Silinske, Marquetto, Grohmann, Battistella e Madruga (2014), Szabo, Costa e Ribeiro (2014).

\begin{tabular}{|l|c|c|c|}
\hline & & & \\
Revistas & Artigos & Interdisciplinar & Administração \\
\hline Revista de Economia e Sociologia Rural (Impresso) & 4 & $\mathrm{~B} 1$ & $\mathrm{~B} 1$ \\
\hline Ciência Rural (UFSM. Impresso) & 3 & $\mathrm{~A} 2$ & $\mathrm{~A} 2$ \\
\hline Ambiente \& Sociedade (Online) & 3 & $\mathrm{~B} 1$ & $\mathrm{~B} 1$ \\
\hline Engenharia Agrícola (Impresso) & 2 & $\mathrm{~B} 1$ & $\mathrm{~A} 2$ \\
\hline Engenharia Agrícola (online) & 2 & $\mathrm{~B} 1$ & $\mathrm{~N} / \mathrm{C}$ \\
\hline Revista Ciência Agronômica (UFC. Online) & 2 & $\mathrm{~B} 1$ & $\mathrm{~N} / \mathrm{C}$ \\
\hline RGSA: Revista de Gestão Social e Ambiental & 2 & $\mathrm{~B} 2$ & $\mathrm{~B} 2$ \\
\hline Acta Amazonica (Impresso) & 1 & $\mathrm{~A} 2$ & $\mathrm{~N} / \mathrm{C}$ \\
\hline Anais da Academia Brasileira de Ciências & 1 & $\mathrm{~A} 1$ & $\mathrm{~N} / \mathrm{C}$ \\
\hline Bragantia (São Paulo, SP. Impresso) & 1 & $\mathrm{~B} 1$ & $\mathrm{~N} / \mathrm{C}$ \\
\hline Cerne (Ufla) & 1 & $\mathrm{~A} 2$ & $\mathrm{~A} 2$ \\
\hline Ciência e Agrotecnologia (Ufla) & 1 & $\mathrm{~A} 2$ & $\mathrm{~A} 2$ \\
\hline Desenvolvimento em Questão & 1 & $\mathrm{~B} 2$ & $\mathrm{~B} 1$ \\
\hline Engenharia Sanitária e Ambiental & 1 & $\mathrm{~A} 2$ & $\mathrm{~A} 2$ \\
\hline Engenharia Sanitária e Ambiental (Online) & 1 & $\mathrm{~B} 1$ & $\mathrm{~N} / \mathrm{C}$ \\
\hline Estudos Avançados (USP. Impresso) & 1 & $\mathrm{~A} 2$ & $\mathrm{~A} 2$ \\
\hline Gestão \& Regionalidade & 1 & $\mathrm{~B} 3$ & $\mathrm{~B} 1$ \\
\hline Pesquisa Agropecuária Tropical (Online) & 1 & $\mathrm{~B} 2$ & $\mathrm{~N} / \mathrm{C}$ \\
\hline Pesquisa Operacional (Impresso) & 1 & $\mathrm{~B} 1$ & $\mathrm{~A} 2$ \\
\hline REAd. Revista Eletrônica de Administração (Porto Alegre. Online) & 1 & $\mathrm{~B} 2$ & $\mathrm{~B} 1$ \\
\hline Recadm : Revista Eletrônica de Ciência Administrativa & 1 & $\mathrm{~B} 2$ & $\mathrm{~B} 2$ \\
\hline Revista Ceres & 1 & $\mathrm{~B} 1$ & $\mathrm{~N} / \mathrm{C}$ \\
\hline Revista de Administração Pública (Impresso) & 1 & $\mathrm{~A} 2$ & $\mathrm{~A} 2$ \\
\hline Scientia Agricola (USP. Impresso) & 1 & $\mathrm{~A} 2$ & $\mathrm{~N} / \mathrm{C}$ \\
\hline Sociedade \& Natureza & 1 & $\mathrm{~B} 1$ & $\mathrm{~N} / \mathrm{C}$ \\
\hline Total Geral & 36 & & \\
\hline Figura 3 Relação de revistas eclassifição & & \\
\hline
\end{tabular}

\section{Figura 3 - Relação de revistas e classificação}

Fonte: Dados da pesquisa (2015)

Na figura 3 é apresentada a relação de revistas com publicações relacionadas ao tema no período analisado, com destaque para a Revista de Economia e Sociologia Rural com quatro publicações relacionadas ao gênero. A Revista Engenharia Agrícola possui artigos nas versões impressa e online que igualmente totalizam quatro publicações relacionadas. Classificando as revistas por estrato da Qualis da Coordenação de Aperfeiçoamento de Pessoal de Nível Superior [Capes] há uma concentração no estrato B1 interdisciplinar, com 18 artigos e A2 Administração com 11 trabalhos.

Nos agrupamentos por palavras-chaves há uma maior referência para as palavras sustentabilidade (12), indicadores de sustentabilidade (5), desenvolvimento sustentável (5), 
agricultura familiar (4), agroecologia (4), eficiência energética (4), gestão ambiental (3), sustentabilidade ambiental (3), agricultura (2), balanço energético (2), eficiência cultural (2), indicadores (2), políticas públicas (2) e sistemas de produção (2). Outras palavras, embora únicas, apresentam pequenas variações, um exemplo é em relação ao "desenvolvimento sustentável" que apresenta variações para desenvolvimento, desenvolvimento rural e desenvolvimento rural sustentável.

Ainda a cultura ou o sistema de produção em que foi aplicado nos artigos podem compor uma palavra-chave como: soja, milho, arroz, bovinocultura, cana-de-açúcar, suinocultura e silagem. Podem também representar a técnica de manejo como: plantio direto, convencional, orgânico ou transgênico.

A palavra-chave "indicadores de sustentabilidade" apresentou variações como: indicadores, indicadores biofísicos e socioeconômicos, indicadores de qualidade do solo, indicadores energéticos. Outros ainda utilizam a palavra-chave para representar a região de estudo, seja o estado, o município, região ou comunidade. Observa-se o fato de a comunidade representar algumas características do estudo, tais como assentamento, assentamentos rurais, comunidades, comunidades rurais ou espaço rural. Por fim, a palavra-chave pode representar o método ou modelo aplicado como Análise Envoltória de Dados (DEA), análise fatorial, análise multivariada, Apoia-NovoRural, balanço energético e dashboard of sustainability.

Em relação às instituições de pesquisa, foi possível vincular 37 instituições diferentes com 54 participações nos 36 artigos, porém, embora possuindo vários autores da mesma instituição, esta foi apenas considerada uma única vez em cada trabalho. A Universidade Federal do Ceará [UFC] contribuiu com seis publicações relacionadas ao tema. A Embrapa com cinco publicações. A Universidade Estadual de São Paulo [Unesp], Universidade Federal do Amazonas [Ufam], Universidade Federal de Campo Grande [Ufcg], Universidade Federal de Lavras [Ufla], Universidade Federal de Minas Gerais [Ufmg], Universidade Federal de Pelotas [UFPel], Universidade Federal de Santa Catarina [Ufsc] e a Universidade de São Paulo [USP] aparecem em duas publicações cada. As demais instituições apresentaram uma publicação.

Nas publicações, a preferência é pelo uso de indicadores, com 21 trabalhos contra 15 que utilizaram índice. As pesquisas de Romanelli e Milan (2005), Campos, Saglietti, Campos e Bueno (2005), Jasper, Biaggioni, Silva, Seki e Bueno (2010), Araujo, Brandão Junior e Colen (2013), Capellesco e Cazella (2013), Ferreira, Neumann e Hoffmann (2014) e Ramos, Campos, Júnior e Silva (2014) concentraram-se no uso de indicadores na análise do sistema produtivo pelo sistema energético, totalizando sete artigos. A análise energética contribui na gestão do conhecimento em agronegócio, sendo um ótimo indicador de desempenho, constituindo-se um instrumento auxiliar para a avaliação da sustentabilidade (Ramos, Campos, Junior, Silva, 2014). A análise energética tem seu foco no sistema produtivo rural. Já Eurich, Weirich Neto e Rocha (2013) utilizaram de índice energético e econômico para análise da sustentabilidade da produção leiteira, em que a análise energética favorável representa um indicador de sustentabilidade positivo.

O sistema produtivo e as comunidades também podem ser analisados por indicadores nos pilares da sustentabilidade, ou seja, nas dimensões econômica, ambiental e social. Os estudos com aplicação em comunidades somam 17 artigos, nove utilizando índices, sendo Barreto, Khan e Lima (2005), Andrade (2007), Neto, Andrade, Rosa, Mota e Lopes (2008), Gomes, Mello e Mangabeira (2009), Damasceno, Kahn e Lima (2011), Alves e Bastos (2011), Rodrigues e Najberg (2012), Santos e Cândido (2013) e Cardoso, Souza, Paiva, Momo e Cunha (2014) e oito trabalhos com uso de indicadores: Finatto e Salamoni (2008), Pereira, Lino, Buschinelli, Barroes e Rodrigues (2010), Meireles, Araujo Neto e Oliveira (2011), Lima, Doria e Freitas (2012), Silva, Barbosa e Albuquerque (2013), Santos, Siqueira, Araújo e Maia (2014), Vidal e Santos (2014) e Carvalho e Oliveira (2010), este último com foco na dimensão econômica.

Os estudos de análise do sistema produtivo com a utilização das dimensões da sustentabilidade somam 10 artigos: Ribas, Severo e Miguel (2007), Araujo e Sá (2008), este abordando principalmente as características socioeconômicas, Souza, Andrade e Cândido (2008), 
Vernetti Junior, Gomes e Schuch (2009), Pugliesi, Marinho, Marques e Lucarelli (2011), este abordando a perda econômica com a erosão, Santos, Mota, Santos, Monteiro e Fontenele (2011), Poerch, Milach, Cavalli, Wasielesky Jr, Möller e Castello (2013), Rodrigues Filho e Juliani (2013), Baretta, Bartz, Fachini, Anselmi, Zortéa e Baretta (2014) e Gomes, Peruzatto, Santos e Sellitto (2014).

Os indicadores e índices podem estar inseridos em modelos para análise ou avaliação da sustentabilidade, totalizando cinco artigos: Pereira, Lino, Buschinelli, Barros, Rodrigues (2010), que utilizaram do Apoia-NovoRural proposto por Rodrigues e Campanhola (2003), e no estudo de Borges e Borges (2014), que aplicaram o modelo Pressão-Estado-Resposta (PER). Já Gomes, Peruzatto, Santos e Sellitto (2014) utilizaram do Sistema de Indicadores de Sustentabilidade da Suinocultura (Siss), adaptado de método Mais de Oliveira (2002). Rodrigues Filho e Juliani (2013) utilizaram o Sistema de Avaliação da Sustentabilidade (SAS) e Rodrigues e Najberg (2012) utilizaram do Dashboard of Sustainability.

\section{CONSIDERAÇÕES FINAIS}

O tema sustentabilidade tem crescido consideravelmente nas pesquisas científicas do tipo meta-análise, inseridos em diversos temas, tais como recursos humanos (Freitas, Souza, Teixeira e Jabbour, 2013), vantagem competitiva (Schimitt, Hayde e Dreher, 2013), inovação (Xavier, Lucca, Richter e Wittmann, 2013), gestão de projetos (Rocha, Gomes, Kneipp e Camargo, 2013), e também analisando a evolução do tema sustentabilidade ambiental nos periódicos nacionais (Souza e Ribeiro, 2013). Estudos relacionados à análise da sustentabilidade e sua mensuração também têm contribuído com o estado da arte, como mencionam Van Bellen (2004), Tayra e Ribeiro (2006), Siche, Agostinho, Ortega e Romeiro (2007) e Veiga (2010).

Assim, buscou-se verificar as pesquisas relacionadas à análise da sustentabilidade aplicada no meio rural, limitando-se no acompanhamento de aplicações práticas que buscam mensurar o nível de sustentabilidade de comunidades ou de sistemas produtivos. Observou-se uma maior concentração de trabalhos relacionados ao uso de indicadores, mas com duas linhas de pesquisas distintas: a primeira, com análise emergética para avaliar a sustentabilidade basicamente de um sistema produtivo e seu consumo de recursos. A segunda linha busca avaliar a sustentabilidade de uma comunidade ou de um sistema produtivo sob a ótica das dimensões da sustentabilidade propostas pela Agenda 21, ou seja, econômica, ambiental e social.

Por vezes, as pesquisas analisam parcialmente a sustentabilidade, com foco apenas no econômico e ambiental, em outras recorrem a uma análise mais completa, incluindo questões territoriais, científicas, tecnológicas, políticas e culturais, além de questões agronômicas. Por fim, essas pesquisas por dimensões ocorrem preferencialmente em comunidade rurais com uso de índices. Observa-se ainda uma evolução histórica crescente nas publicações relacionadas à análise da sustentabilidade no meio rural.

Como contribuição para o desenvolvimento de pesquisas na área, este estudo identificou ferramentas de mensuração da sustentabilidade no meio rural, para adaptação às necessidades e aos objetivos de sua aplicação, destacando-se as ferramentas Apoia-NovoRural e o modelo PressãoEstado-Resposta (PER) na análise da sustentabilidade. A composição de um índice para determinação do nível de sustentabilidade em comunidades representa uma proposta interessante, apesar de a atribuição de pesos e a distribuição equilibrada dos indicadores nas fórmulas de cálculo ser uma limitação. Contudo, apresentar um resultado final com as interações de todas as dimensões da sustentabilidade em um único índice pode facilitar a gestão da sustentabilidade. 


\section{REFERÊNCIAS}

Agência Europeia do Ambiente [AEA]. (1995) Europe's Environment: the Dobris Assessment. Agência Europeia do Ambiente, Copenhagen.

Alves, L. B., \& Bastos, R. P. (2011) Sustentabilidade em Silvânia (GO): o caso dos assentamentos rurais São Sebastião da Garganta e João de Deus. Revista de Economia e Sociologia Rural, 49(2), 419-448.

Andrade, A. L. M. D. (2007) Indicadores de sustentabilidade na Reserva de Desenvolvimento Sustentável do Piranha, Manacapuru, Amazonas, Brasil. Acta Amazonica, 37(3), 401-412.

Araújo, J. D., \& Sá, M. D. (2008) Sustentabilidade da piscicultura no baixo São Francisco alagoano: condicionantes socioeconômicos. Ambiente \& Sociedade, 11(2), 405-424.

Araujo, A. V. D., Brandão Junior, D. D. S., \& Colen, F. (2013) Energetic analysis of landrace varieties and hybrids of corn produced in different technological levels of management. Engenharia Agrícola, 33(4), 625-635.

Assad, M. L. L., Almeida, J. (2004) Agricultura e sustentabilidade: contexto, desafios e cenários. Ciência e Ambiente. 29, 15-30. Recuperado em Janeiro, 2015, de http://www.is.cnpm.embrapa.br/bibliografia/2004_AGRICULTURA_E_SUSTENTABILIDADE_ CONTEXTO_DESAFIOS_E_CENARIOS.pdf.

Baretta, D., Bartz, M. L. C., Fachini, I., Anselmi, R., Zortéa, T., \& Baretta, C. R. D. M. (2014) Soil fauna and its relation with environmental variables in soil management systems. Revista Ciência Agronômica, 45(5SPE), 871-879.

Barreto, R. C. S., Khan, A. S., \& Lima, P. V. P. S. (2005) Sustentabilidade dos assentamentos no município de Caucaia-CE. Revista de Economia e Sociologia Rural, 43(2), 225-247.

Berliner, C., \& Brimson, J. A. (1988) Cost management for today's advanced manufacturing: The CAM-I conceptual design. Boston: Harvard Business School Press.

Borges, F. Q., \& Borges, F. Q. (2014) Gestão regional e sustentabilidade energética: uma análise de indicadores no setor agropecuário paraense (2002-2010). Gestão \& Regionalidade, 30(88), 81-95.

Briquel, V., Vilain, L., Bourdais, J. L., Girardin, P., Mouchet, C., \& Viaux, P. (2001) La méthode IDEA (indicateurs de durabilité des exploitations agricoles): une démarche

pédagogique. Ingénieries-EAT, 25, 29-39. Recuperado em Fevereiro, 2015, de https://hal.archivesouvertes.fr/hal-00464508/document.

Bouni, C. (1996) Indicateurs de développement durable: l'enjeu d'organiser une information hétérogène pour préparer une décision multicritère. In: Colloque International, Abbay De Fontevraud-Indicateurs De Développemente Durable, Paris.

Callado, A. L. C., \& Fensterseifer, J. E. (2009) Indicadores de sustentabilidade. In J. L. Albuquerque (org.). Gestão ambiental e responsabilidade social: conceitos, ferramentas e aplicações (213-234). São Paulo: Atlas. 
Campos, A. T., Saglietti, J. R. C., Campos, A. T., \& Bueno. O. C. (2005) Análise energética na produção de feno de Cynodon dactylon (L.) Pers. Engenharia Agrícola, 25(2), 349-358.

Capellesco, A. J., \& Cazella, A. A. (2013) Indicador de sustentabilidade dos agroecossistemas: estudo de caso em áreas de cultivo de milho. Ciência Rural, 43(12), 2297-2303.

Cardoso, B. L. D., de Souza, W. J., de Paiva, J. A., Momo, D. C., \& Cunha, A. S. R. (2014)

Desenvolvimento territorial sustentável: estudo comparativo de indicadores do sistema de gestão estratégica em territórios rurais do Rio Grande do Norte. Revista Eletrônica de Ciência Administrativa-Recadm, 13(1), 39-55.

Carvalho, P. G. M., \& Barcellos, F. C. (2009) Construindo indicadores de sustentabilidade. Indicadores Econômicos FEE, 37(1). Recuperado em Fevereiro, 2015, de http://revistas.fee.tche.br/index.php/indicadores/article/viewArticle/2280.

Carvalho, R. S., \& Oliveira, A. D. (2010). Economic feasibility of timber management in extractive settlement projects of southwestern Amazonia. Cerne, 16(4), 505-516.

Comissão de Políticas de Desenvolvimento Sustentável e da Agenda 21 Nacional [CPDS]. (2004). Agenda 21 brasileira: ações prioritárias. Brasília: Ministério do Meio Ambiente. Recuperado em Janeiro, 2015, de http://www.mma.gov.br/estruturas/agenda21/_arquivos/acoes2edicao.pdf.

Cooper, H. M., \& Lindsay, J. J. (1998) Research synthesis and meta-analysis. In: L. Bickman \& D. J. Rog (Eds.) Handbook of applied social research methods (315-342). Thousand Oaks, CA: Sage Publications.

Costa, A. A. V. M. R. (2010) Agricultura sustentável III: Indicadores. Revista de Ciências Agrárias, 33(2), 90-105. Recuperado em Janeiro, 2015, de http://www.scielo.oces.mctes.pt/scielo.php?script=sci_arttext\&pid=S0871018X2010000200009

Damasceno, N. P., Khan, A. S., \& Lima, P. V. P. S. (2011) O impacto do Pronaf sobre a sustentabilidade da agricultura familiar, geração de emprego e renda no Estado do Ceará. Revista de Economia e Sociologia Rural, 49(1), 129-156.

Eurich, J., Neto, P. H. W., \& Rocha, C. H. (2013) Índices emergéticos de sustentabilidade da produção leiteira em uma propriedade de base familiar em Palmeira, Paraná, Brasil. Revista Ceres, 60(3), 332-338.

Ferreira, F. F., Neumann, P. S., \& Hoffmann, R. (2014) Análise da matriz energética e econômica das culturas de arroz, soja e trigo em sistemas de produção tecnificados no Rio Grande do Sul. Ciência Rural, 44(2), 380-385.

Finatto, R. A., \& Salamoni, G. (2008) Agricultura familiar e agroecologia: perfil da produção de base agroecológica do município de Pelotas/RS. Sociedade \& Natureza, 20(2), 199-217.

Freitas, W. R. S., de Souza, M. T. S., Teixeira, A. A., \& Jabbour, C. J. C. (2013) Produção científica sobre gestão de recursos humanos e sustentabilidade: síntese e agenda de pesquisa. Revista de Ciências da Administração, 15(36), 11-28.

Gil, A. C. (2010) Como elaborar projetos de pesquisa. (5a. ed.). São Paulo: Atlas. 
Gomes, E. G., Mello, J. C. C. B. S., \& Mangabeira, J. A. D. C. (2009) Estudo da sustentabilidade agrícola em município amazônico com análise envoltória de dados. Pesquisa Operacional, 29(1), 23-42.

Gomes, L. P., Peruzatto, M., Santos, V. S. D., \& Sellitto, M. A. (2014) Indicadores de sustentabilidade na avaliação de granjas suinícolas. Eng. sanit. ambient, 19(2), 143-15.

Hardi, P., Zdan, T. (1997) Assessing sustainable development: principles in practice. Winnipeg: International Institute for Sustainable Development. Recuperado em Fevereiro, 2015, de http://www.iisd.org/pdf/bellagio.pdf.

Hardi, P., Zdan, T. J. (2000) The dashboard of sustainability. Winnipeg: International Institute for Sustainable Development [IISD].

Hopwood, B., Mellor, M., \& O'Brien, G. (2005) Sustainable development: mapping different approaches. Sustainable development, 13(1), 38-52.

Jasper, S. P., Biaggioni, M. A., Silva, P. R., Seki, A. S., \& Bueno, O. C. (2010) Análise Energética da cultura do crambe (Crambe abyssinica hochst) produzida em plantio direto. Engenharia Agrícola, 30(3), 395-403.

Kummer, L. (2007) Metodologia participativa no meio rural: uma visão interdisciplinar. conceitos, ferramentas e vivências. Salvador: GTZ.

Lima, M. A. L., Doria, C. R. D. C., \& Freitas, C. E. D. C. (2012) Pescarias artesanais em comunidades ribeirinhas na amazônia brasileira: perfil socioeconômico, conflitos e cenário da atividade. Ambiente \& Sociedade, 15(2), 73-90.

Luca, M. M. M., Cardoso, V. I. C, Vasconcelos, A. C., \& Pontes, A. B. (2014) Análise da produção científica referente à temática de sustentabilidade em pesquisas da Administração. RAE, Rio de Janeiro, 15(3), 469-500.

Masera, O., Astier, M., \& López-Ridaura, S. (1999) Sustentabilidad y manejo de recursos naturales: el marco de evaluación Mesmis. Mexico: Mundi-prensa.

Meireles, A. C. M., Araujo Neto, J. R., \& Oliveira, L. J. (2011) Sustentabilidade do modelo agrícola da bacia do riacho Faé. Revista Ciência Agronômica, 42(1), 84-91.

Melo, P. L. R., \& Andreassi, T. (2010) Publicação científica nacional e internacional sobre franchising: Levantamento e análise do período 1998-2007. RAC-Revista de Administração Contemporânea, 14(2), 268-288.

Neely, A., Richards, H., Mills, J., Platts, K., \& Bourne, M. (1997) Designing performance measures: a structured approach. International journal of operations \& Production management, 17(11), 1131-1152.

Neto, J. A. C., Andrade, E. M., Freitas Rosa, M., Mota, F. S. B., \& Lopes, J. F. B. (2008) Índice de sustentabilidade agroambiental para o perímetro irrigado Ayres de Souza. Ciência e Agrotecnologia, 32(4), 1272-1279. 
Organization for Economic Cooperation and Development [OCDE] (1993) OECD core set of indicators for environmental performance reviews: A synthesis report by the Group on the State of the Environment. Environment monographs, 83. Paris: Organization for Economic Cooperation and Development.

Oliveira, J. H. R. (2002) M.A.I.S. Método para avaliação de indicadores de sustentabilidade organizacional. Tese (Doutorado), Programa de Pós-graduação em Engenharia de Produção, Universidade Federal de Santa Catarina, Florianópolis.

Pereira, J. M., Lino, J. S., Buschinelli, C. C. A., Barros, I., \& Rodrigues, G. S. (2010) Integrated farm environmental management and biodiversity conservation: a case study in the caratinga biological station (Minas Gerais State, Brazil). Pesquisa Agropecuária Tropical, 40(4), 401-413

Pugliesi, A. C. V., Marinho, M. D. A., Marques, J. F., \& Lucarelli, J. R. F. (2011) Valoração econômica do efeito da erosão em sistemas de manejo do solo empregando o método custo de reposição. Bragantia, 70(1), 113-121.

Raupp, F. M., \& Beuren, I. M. (2012) Metodologia da pesquisa aplicável às ciências sociais. In I. M. Beuren (Org). Como elaborar trabalhos monográficos em contabilidade: teoria e prática (7697). (3a. ed.). São Paulo: Atlas.

Ramos, M. C., Campos, A. T., Junior, T. Y., Silva, K. C. P. (2014) Sustentabilidade na produção de leite: balanço energético em sistema intensivo de produção com visão focada nos processos. Engenharia Agrícola, 34(3), 473-484.

Ribas, R. P., Severo, C. M., \& Miguel, L. D. A. (2007) Agricultura familiar, extrativismo e sustentabilidade: o caso dos" samambaieiros" do litoral norte do Rio Grande do Sul. Revista de Economia e Sociologia Rural, 45(1), 205-226.

Rocha, A. C., Gomes, C. M., Kneipp, J. M., \& Camargo, C. R. (2013) Gestão de projetos e sustentabilidade: um estudo bibliométrico da produção científica na Base Web of Science. Revista de Gestão e Projetos-GeP, 4(3), 73-97.

Rodrigues, G. S., \& Campanhola, C. (2003) Sistema integrado de avaliação de impacto ambiental aplicado a atividades do Novo Rural. Pesquisa Agropecuária Brasileira, 38(4), 445-451.

Rodrigues, D. M., \& Najberg, E. (2012) Indicadores de sustentabilidade das políticas públicas decorrentes da expansão do setor sucroalcooleiro em Carmo do Rio Verde (GO). Revista de Gestão Social e Ambiental, 6(3), 61-77.

Rodrigues Filho, S., \& Juliani, A. J. (2013) Sustentabilidade da produção de etanol de cana-deaçúcar no Estado de São Paulo. Estudos Avançados, 27(78), 195-212.

Romanelli, T. L., \& Milan, M. (2005) Energy balance methodology and modeling of supplementary forage production for cattle in Brazil. Scientia Agricola, 62(1), 1-7.

Sanches, G. F., \& Matos, M. M. (2012) Marcos metodológicos para sistematização de indicadores de sustentabilidade da agricultura. (Syn) thesis, 5(2), 255-267.

Santos, J. G., \& Cândido, G. A. (2013) Sustentabilidade e agricultura familiar: um estudo de caso em uma associação de agricultores rurais. Revista de Gestão Social e Ambiental, 7(1), 70-86. 
Santos, E. S., Mota, S., Santos, A. B., Monteiro, C. A. B., \& Fontenele, R. M. M. (2011) Avaliação da sustentabilidade ambiental do uso de esgoto doméstico tratado na piscicultura. Eng Sanit Ambient, 16(1), 45-54.

Santos, C. F., Siqueira, E. S., Araújo, I. T., \& Maia, Z. M. G. (2014) A agroecologia como perspectiva de sustentabilidade na agricultura familiar. Ambiente \& Sociedade, 17(2), 33-52.

Siche, R., Agostinho, F., Ortega, E., \& Romeiro, A. (2007) Índices versus indicadores: precisões conceituais na discussão da sustentabilidade de países. Ambiente \& sociedade, 10(2), 137-148.

Silinske, J., Marquetto, M. F., Grohmann, M. Z., Battistella, L. F., \& Madruga, L. R. D. R. G. (2014) Estudo bibliométrico sobre a sustentabilidade na área de economia empresarial. Amazônia, Organizações e Sustentabilidade, 3(2), 101-120.

Silva, A. R. P., Barbosa, M. J. D. S., \& Albuquerque, F. D. S. (2013) Sustentabilidade de empreendimentos econômicos solidários: análise da Cooperativa dos Fruticultores de Abaetetuba.. RAP: Revista Brasileira de Administração Pública, 47(5), 1189-1211.

Souza, E. G., Andrade, E. O., \& Cândido, G. A. (2008) A aplicação das dimensões do desenvolvimento sustentável: um estudo exploratório nos municípios produtores de leite bovino no Estado da Paraíba. Revista Eletrônica de Administração, 14(3), 650-669.

Souza, M. D., \& Ribeiro, H. C. M. (2013) Sustentabilidade ambiental: uma meta-análise da produção brasileira em periódicos de administração. $R A C, 17(3), 368-396$.

Szabo, V., Costa, B. K., \& Ribeiro, H. C. M. (2014) Stakeholders e sustentabilidade: produção científica internacional e nacional entre 1998 e 2011. Revista Brasileira de Estratégia, 7(2), 174190.

Tayra, F., \& Ribeiro, H. (2006) Modelos de indicadores de sustentabilidade: síntese e avaliação crítica das principais experiências. Saúde e Sociedade, 15(1), 84-95.

Tironi, L. F., Silva, L. C. E., Vianna, S. M., \& Médici, A. C. (1991) Critérios para geração de indicadores de qualidade e produtividade no serviço público. Brasília: Ipea/Mefp. Recuperado em Fevereiro, 2015, de http://hdl.handle.net/11058/1701.

Van Bellen, H. M. (2004). Indicadores de sustentabilidade: um levantamento dos principais sistemas de avaliação. Cadernos Ebape.BR, 2(1), 01-14.

Veiga, J. E. D. (2010) Indicadores de sustentabilidade. Estudos avançados, 24(68), 39-52.

Vernetti Junior, F. D. J., Gomes, A. S., \& Schuch, L. O. B. (2009) Sustentabilidade de sistemas de rotação e sucessão de culturas em solos de várzea no Sul do Brasil. Ciência Rural, 39(6), 17081714.

Vidal, D. L., \& Santos, D. P. A. (2014) Sustentabilidade rural no Semiárido Cearense: uma aproximação social, biofísica e microeconômica em comunidades rurais. Desenvolvimento em Questão, 12(28), 170-192. 
Xavier, T. R., Lucca, C., Richter, A. S., \& Wittmann, M. L. (2013) Sustainability and innovation: a bibliometric study about the international publications. Revista Eletrônica de Estratégia \& Negócios, 6(3), 27-49.

Data da submissão: 27/01/2016

Data da publicação: 30/04/2016 